Medicine Updates Faculty of medicine July 2021,volume 6, issue 6

\title{
$10^{\mathrm{TH}}$
}

https://muj.journals.ekb.egdean@med.psu.edu.eg vice_dean_postgraduate@med.psu.edu.eg DOI: 10.21608/MUJ.2021.181019

Pages:116 - 126

\section{SPRING CONGRESS OF}

\section{THE EGYPTIAN ORTHOPAEDIC ASSOCIATION}

\author{
IN COLLABORATION WITH
}

\section{FACULTY OF MEDICINE PORT SAID UNIVERSITY}

\section{8-9 APRIL 2021}
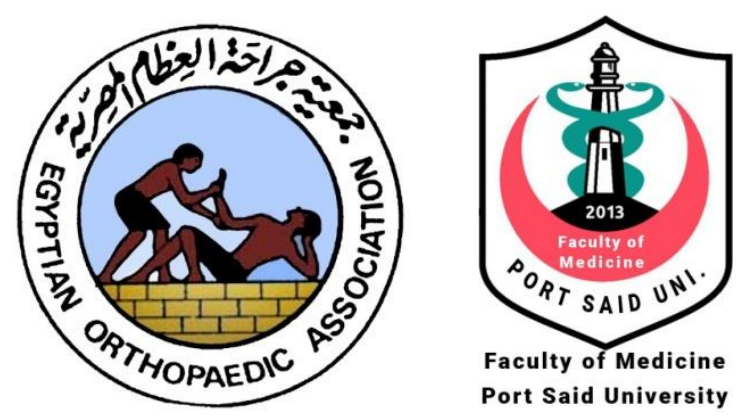

PORTO SAID Hotel- PORTSAID, Egypt . 
Dear Colleagues:

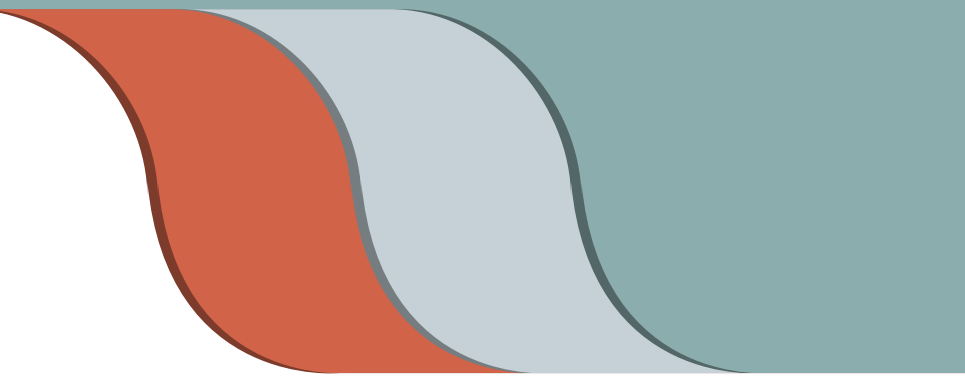

On behalf of the Egyptian Orthopaedic Association, it's our great pleasure to welcome you all to Port Said, The $10^{\text {th }}$ Spring Congress of The Egyptian Orthopaedic Association In Collaboration with Faculty of Medicine- Port Said University.

The congress this year has been designed to provide an innovative and comprehensive overview of the latest research developments on "Upper Limb Trauma and Sports Medicine ", many distinguished Professors are taking part in all symposia that will include super scientific material that was carefully selected to highlight the featured topics of our congress agenda this year.

We hope that you will enjoy this congress and we hope it will stimulate creative exchange of ideas and will be personally rewarding.

Congress Board 


\title{
Congress Board
}

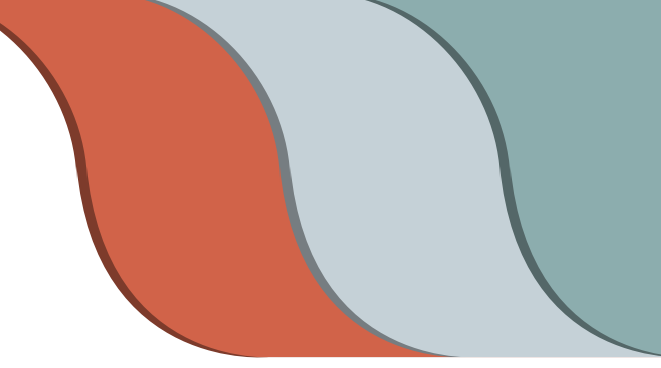

General Secretary of Congress

Prof. Khaled Sabry

Chairman of scientific committee

\author{
Prof. Alaa El Zoheiry
}

Organizing Committee

Dr Yasser Saqr - Dr Mohamed Abdel Aziz Sabry

Dr Ashraf Abdel Aziz - Dr Ahmed Toreh

Treasurer of EOA

Prof. Hani El Mowafi

General Secretary of EOA

Prof. Abdel Salam Eid

President of EOA

Prof. Gamal Hosny 
THURSDAY $8^{T H}$ APRIL

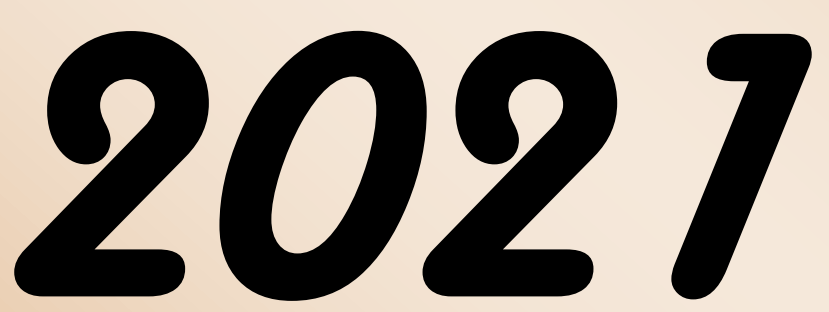




\section{Thursday 8, APRIL 2021}

\begin{tabular}{lll} 
12:00-01:00 & \multicolumn{1}{c}{ Session1: SPORTS MEDICENE } \\
Chairmen & $\begin{array}{l}\text { Prof. Ahmed Mahrous } \\
\text { Prof. Khaled Sabry } \\
\text { Prof. Adel EIShamy }\end{array}$ & \\
\hline $12: 00$ & $\begin{array}{l}\text { Arthroscopic substalar Arthrodesis } \\
\text { Yasser Saqr }\end{array}$ & Egypt \\
\hline $12: 15$ & $\begin{array}{l}\text { Latarjet Procedure In Shoulder instability } \\
\text { Ahmed Toreh }\end{array}$ & Egypt \\
\hline $12: 30$ & $\begin{array}{l}\text { Management of Massive RC Tear } \\
\text { Hossam El Begawi }\end{array}$ & \\
\hline $12: 45$ & Sports Medicine vs Covid -19 & Egypt \\
\hline
\end{tabular}

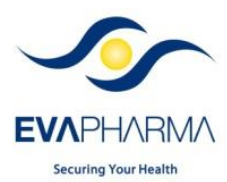




\section{Thursday 8, APRIL 2021}

\begin{tabular}{|c|c|c|}
\hline 03:00-04:30 & Session2: FOOT \& ANKLE & \\
\hline Chairmen & $\begin{array}{l}\text { Prof. Ahmed Kholief } \\
\text { Prof. Hani El Mowafi } \\
\text { Prof. Sherif Sokar }\end{array}$ & \\
\hline \multirow[b]{2}{*}{ 03:00 } & Lateral ligament injury & Egypt \\
\hline & Hani El Mowafi & \\
\hline \multirow{2}{*}{ 03:15 } & Osteochondral injury & Egypt \\
\hline & Ahmed Kholief & \\
\hline \multirow[b]{2}{*}{ 03:30 } & Acute Achilles' tendon rupture & Egypt \\
\hline & Mohamed Mokhtar & \\
\hline \multirow{2}{*}{ 03:45 } & Syndesmosis injury & Egypt \\
\hline & Amr Farouk & \\
\hline 04:00 & $\begin{array}{l}\text { Sanofi symposium } \\
\text { VTE management in orthopedic } \\
\text { Prof. Essam Elsherif }\end{array}$ & \\
\hline 04:30 & Change Break & \\
\hline
\end{tabular}




\section{Thursday 8, APRIL 2021}

\begin{tabular}{lll} 
04:30-06:00 & \multicolumn{1}{c}{ Session3: Sports Medicine } & \\
Chairmen & $\begin{array}{l}\text { Prof. Adel Adawy } \\
\text { Prof. Bahaa Koranah } \\
\text { Prof. Essam El Karef } \\
\text { Lumbar Disc Prolapse } \\
\text { Mohamed Wafa }\end{array}$ & Egypt \\
\hline $04: 30$ & $\begin{array}{l}\text { Limits of SAD } \\
\text { Yasser Saqr }\end{array}$ & Egypt \\
\hline $04: 55$ & $\begin{array}{l}\text { O.C.D Knee } \\
\text { Abdel Samie Halawa }\end{array}$ & Egypt \\
\hline $05: 05$ & $\begin{array}{l}\text { Aerosol generating procedures in trauma and orthopaedic } \\
\text { in the era of the Covid-19 pandemic; What do we know? }\end{array}$ & Egypt \\
\hline $05: 15$ & Karim Mehana & \\
\hline $05: 25$ & \multicolumn{1}{c}{ Amgen Symposium } \\
\hline $06: 00$ & \multicolumn{2}{c}{ End Of the day } \\
\hline
\end{tabular}

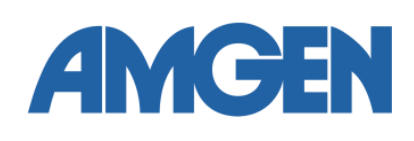




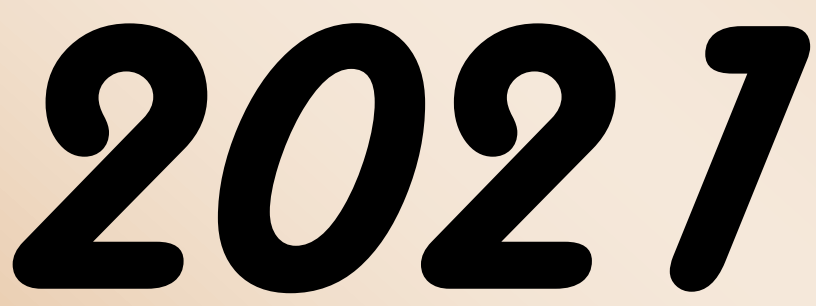




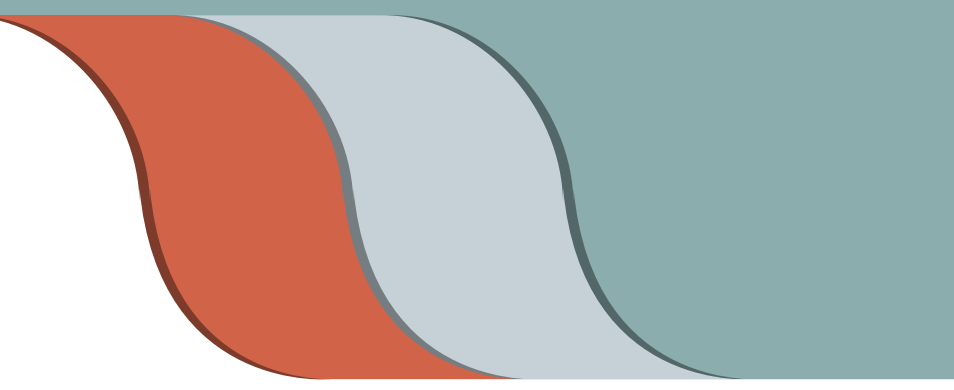

\section{Friday 9, APRIL 2021}

\begin{tabular}{|c|l|c|}
\hline 09:00-10:30 & \multicolumn{1}{|c|}{ Session 4: Miscellaneous } & \\
\hline Chairmen & $\begin{array}{l}\text { Prof. Abdel Salam Eid } \\
\text { Prof. Mohamed Goda } \\
\text { Prof. Mohamed Radwan }\end{array}$ & Egypt \\
\hline $09: 00$ & $\begin{array}{l}\text { Respect bone otherwise infection fires. case presentation } \\
\text { Raouf El Nagar }\end{array}$ & Egypt \\
\hline $09: 10$ & $\begin{array}{l}\text { Two llizarv rings versus four ilizarov rings in all schanz screw } \\
\text { fixation of comminuted fractures of the tibia, Experimental } \\
\text { mechanical testing } \\
\text { Mohamed Abdel-Wahed Kotb }\end{array}$ & Egypt \\
\hline $09: 20$ & $\begin{array}{l}\text { Management of Bone defect in forearm In young child } \\
\text { Adel Khamis }\end{array}$ & Egypt \\
\hline $09: 30$ & $\begin{array}{l}\text { Nonunion humerus, llizarov principles approach } \\
\text { Mohamed Fadel }\end{array}$ & \\
\hline $09: 40$ & $\begin{array}{l}\text { Treatment of ununited humerus } \\
\text { Mohamed Abdel Aal Hussien }\end{array}$ \\
\hline $09: 50$ & \multicolumn{1}{|c|}{ MSD SYMScussion } \\
\hline $10: 00$ & \multicolumn{1}{|c|}{} \\
\hline
\end{tabular}




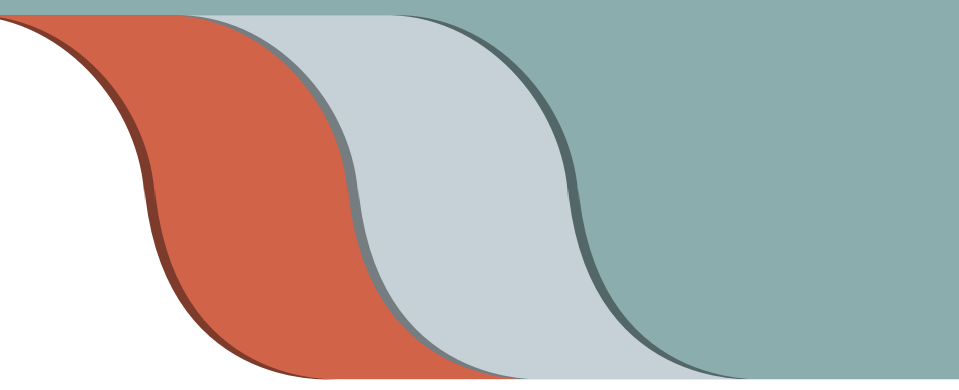

\section{Friday 9, APRIL 2021}

\begin{tabular}{|c|c|c|}
\hline 10:30-12:00 & Session5: Upper Limb & \\
\hline Chairmen & $\begin{array}{l}\text { Prof. Hassan El Zaher } \\
\text { Prof. Hazem Abdel Azeem } \\
\text { Prof. Mohamed Al Ashhab }\end{array}$ & \\
\hline \multirow[b]{2}{*}{ 10:30 } & Implant choice in distal radius fracture & Egypt \\
\hline & Abdel Hakim Abdullah & \\
\hline \multirow{2}{*}{ 10:45 } & Post- traumatic Elbow Instability & Egypt \\
\hline & Ashraf El Tabie & \\
\hline \multirow{2}{*}{ 11:00 } & Fractures of the proximal humerus, an overview. & Egypt \\
\hline & Abdel Salam Eid & \\
\hline \multirow[b]{2}{*}{ 11:00 } & Injuries of Long Head of Biceps Tendon & Egypt \\
\hline & Amr Ahmed & \\
\hline \multirow{2}{*}{$11: 15$} & $\begin{array}{l}\text { Management of complex elbow dislocation with } \\
\text { multifragmentary Head radius }\end{array}$ & Egypt \\
\hline & Mahmoud M Hussien & \\
\hline 11:30 & Change Break \& Gomaa Prayer & \\
\hline
\end{tabular}




\section{Friday 9, APRIL 2021}

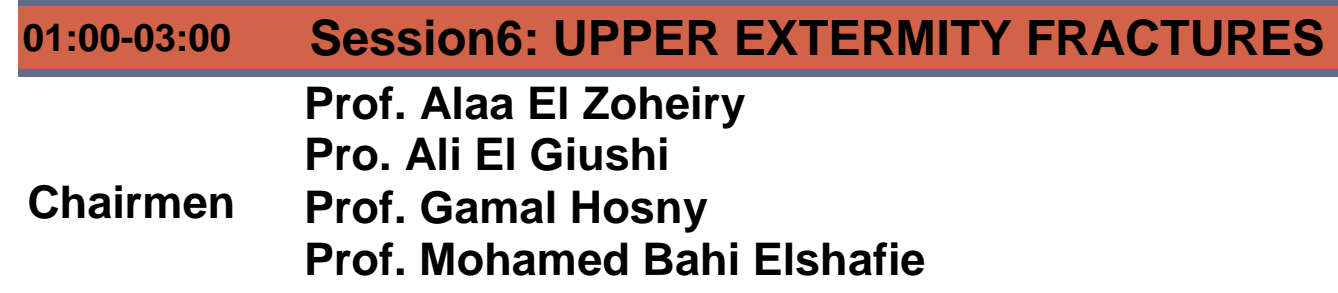

Alaa El Zoheiry

Egypt

New trends for treatment of open fractures

Gamal Hosny

Egypt

Upper extremity fractures, role of external fixation

Mohamed Bahi Elshafie

Egypt

Biomechanics of shoulder joint

CASE DISCUSSION (Mohamed Zaki)

03:00

UTOPIA SYMPOSIUM (MEET THE MASTER)

03:30

Closing Awards 